\title{
Aspectos de diseño y seguridad en apartaderos de túneles de carretera
}

\author{
Design and safety issues in lay-bys of road tunnels
}

Fecha de entrega: 29 de mayo 2015

Fecha de aceptación: 2 de noviembre 2015

\section{Fernando Hacar, Jimmy Jönsson y Gabriele Vigne}

JVVA Fire \& Risk, Velázquez 157, Ibercenter, 28002 Madrid, España, fernando.hacar@jvvafire.com, jimmy.jonsson@jvvafire.com, gabriele.vigne@jvvafire.com

Los apartaderos o lay-bys en túneles de carretera se construyen para ser empleados durante la explotación del túnel, tanto por los usuarios en caso de emergencia como por los servicios de explotación. Según la normativa específica de cada país, a partir de cierta longitud del túnel, entre otros factores, se han de construir dichos apartaderos a determinadas distancias entre ellos. La geometría de los mismos presenta alguna diferencia entre las diferentes normativas. Se exponen algunas de dichas normativas. Los apartaderos también presentan algunos aspectos sobre los que se reflexiona, comenzando para ello con el conocimiento de accidentes habidos en diversos túneles por colisión frontal, o más o menos descentrada, de vehículos ligeros y pesados contra el paramento final de los apartaderos. Las soluciones para mitigar los efectos de ese tipo de colisiones, muy frecuentemente con graves consecuencias para los ocupantes de los vehículos, han de ser objeto de estudios que contemplen ensayos fisicos que validen los sistema de protección que se instalen en los túneles.

Palabras clave: túnel, accidentes, colisión, apartaderos
Lay-bys in road tunnels are built to be used during tunnel operation, both for users in case of emergency and for operating services. According to the specific regulations of each country, after a certain length of the tunnel, among other factors, those lay-bys need to be built at certain distances between them. The geometry of the lay-bys presents differences depending on the different regulations. Some of these regulations are presented in this article. Lay-bys also present some issues that need special attention, like the knowledge of accidents that have occurred in various tunnels due to frontal collisions, more or less offset, of light and heavy vehicles against the facing end of the lay-bys. The solutions to mitigate the effects of such collisions, very often with serious consequences for the occupants of the vehicles, need be addressed through physical testing that can validate the protection systems to be installed in the tunnels.

Keywords: tunnel, accidents, crash, lay-bys

\section{Introducción}

Muchos túneles no disponen de carriles de emergencia o arcenes suficientemente anchos -de más de $2.5 \mathrm{~m}$ - para los vehículos que por alguna razón se vean obligados a detenerse dentro del mismo. Los apartaderos (anchurones para aparcamiento, lay-bys, emergency stop) permiten que los vehículos se detengan en un túnel sin bloquear el carril de circulación, reduciendo las perturbaciones al tráfico y el riesgo de colisión (Figura 1a). Es más seguro para los ocupantes del vehículo detenido salir del mismo en un apartadero, y especialmente para las personas discapacitadas, para, por ejemplo, usar un teléfono de emergencia (Figura 1b). Igualmente son utilizados para labores de mantenimiento del túnel ya que garantizan un estacionamiento seguro para los vehículos.
Para definir geométricamente la sección interior de un túnel se deben dibujar las diferentes secciones correspondientes a los diferentes peraltes en ambos sentidos de las curvas, esto es, en ambos signos del peralte, y trazar la curva óptima que satisfaga a todas esas diferentes secciones. Normalmente se define una única sección tipo interior final para el túnel acabado con las diferencias debidas a los diferentes peraltes, exceptuando zonas concretas, como los apartaderos cuya sección debe ser estudiada de forma específica. En esos estudios para la definición de la sección del túnel se han de considerar también los servicios que puedan ser necesarios instalar, como por ejemplo ventilación, conducciones bajo calzada, caces para la evacuación de vertidos, arquetas, pozos de registro, así como la posibilidad de tener que emplear un tipo o más de sostenimientos y contrabóvedas (Figura 2). 

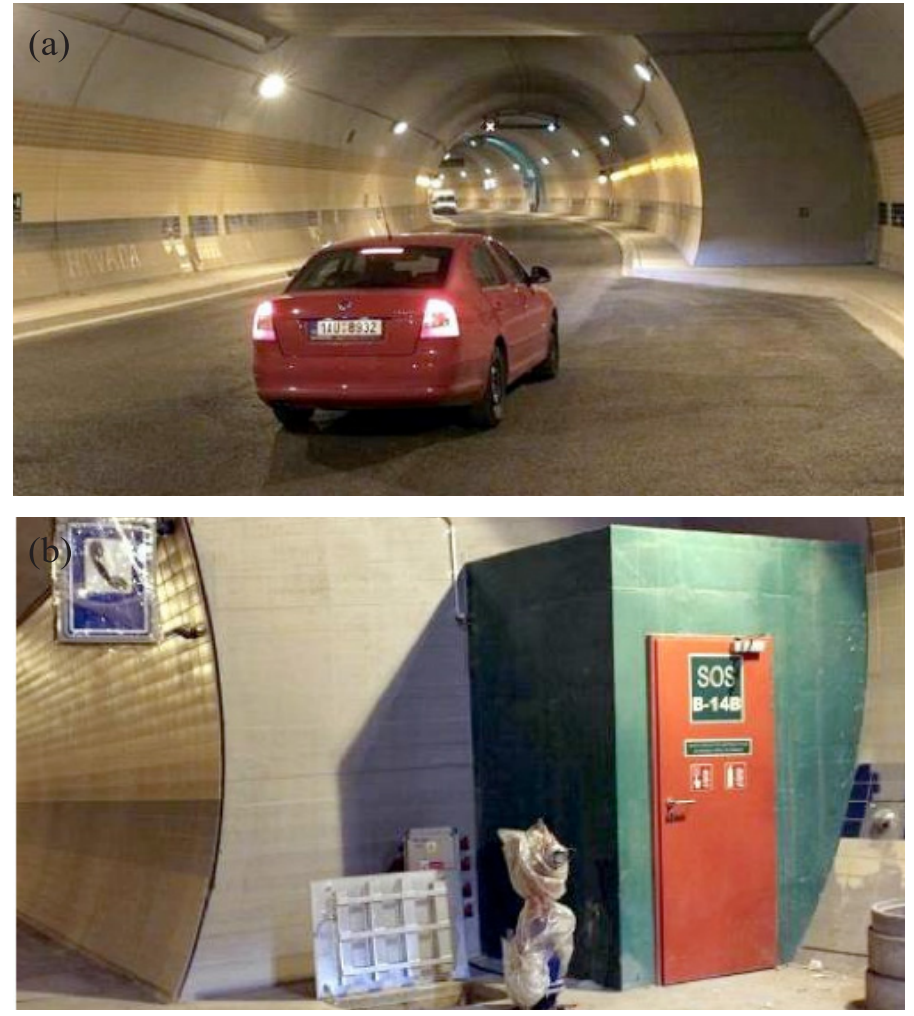

Figura 1: Apartaderos en túnel Blanka, Praga (MAFRA, 2014)

(a)



(b)

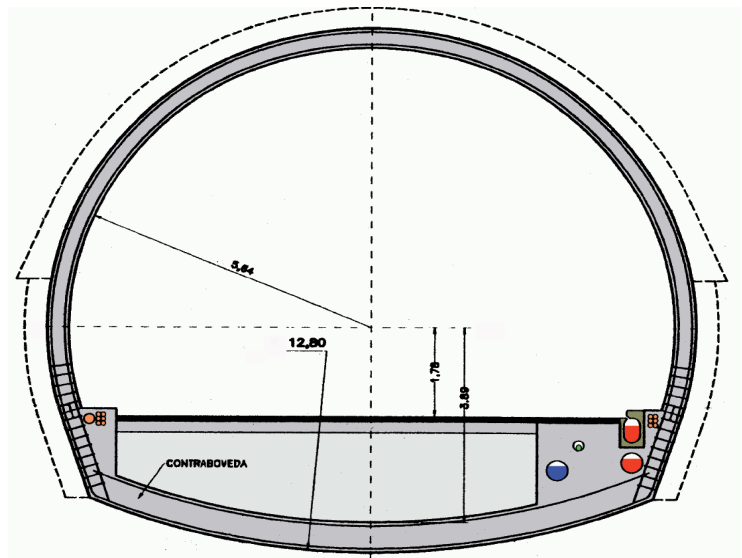

Figura 2: Ejemplo de dos secciones definidas para un mismo túnel, a) sección tipo ordinaria y b) sección tipo de un tramo con contrabóveda
La definición de una sección diferente a la del resto del túnel para los apartaderos obligará a un sistema específico de construcción, y si se emplean carros de encofrados para realizar el revestimiento definitivo, éstos deberán estar especialmente diseñados para permitir el trabajar con esa sección diferente del túnel (Figura 3). Aunque no siempre se emplean pues puede realizarse el hormigonado por sistemas tradicionales de encofrado.

(a)

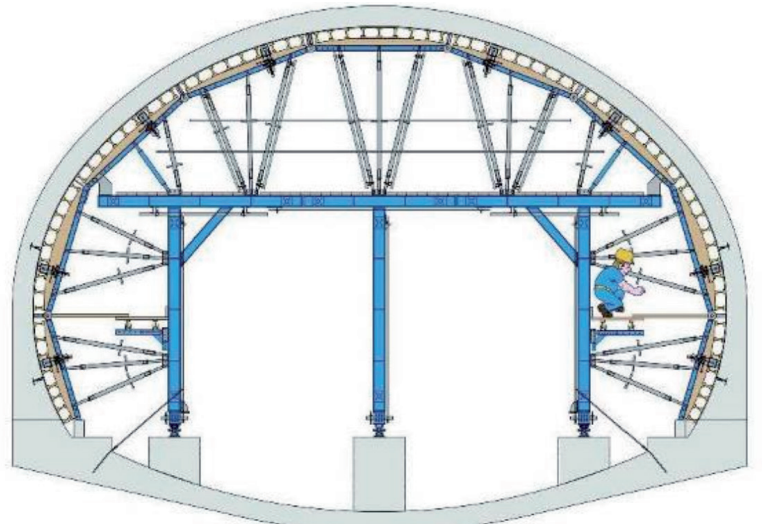

(b)

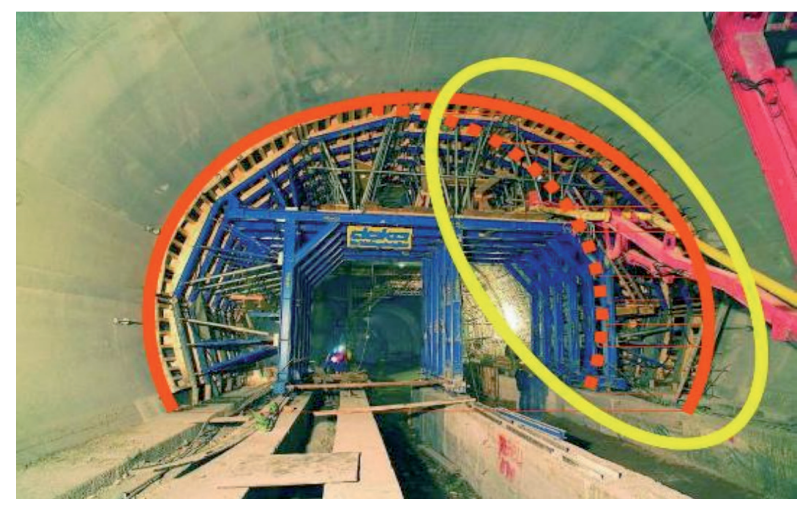

Figura 3: a) Encofrado para los apartaderos y b) túnel de Javorova, autopista A-6, Croacia (DOKA, 2007)

Como otro ejemplo, la Figura 4 muestra una sección en el túnel de Marcavalle, Perú.

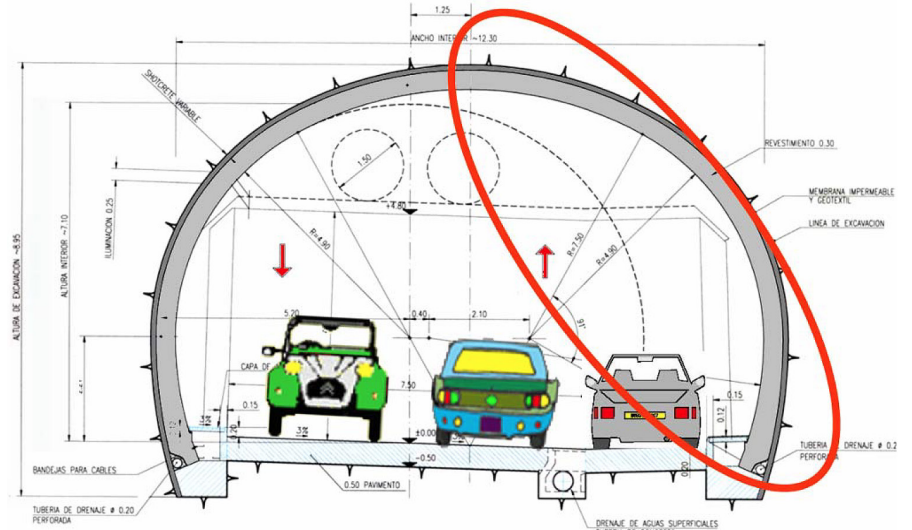

Figura 4: Sección en zonas de apartaderos, túnel Marcavalle, Oroya, Perú (Samaniego et al., 2008) 
Normativa y estudios relativo a apartaderos

\section{Dossier Pilote des Tunnels}

En el Dossier Pilote des Tunnels (CETU, 1976) se propone para los apartaderos la geometría mostrada en la Figura 5.

(a)

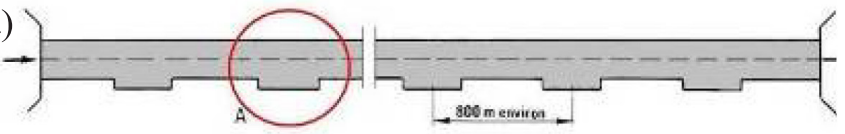

(a)

(b)

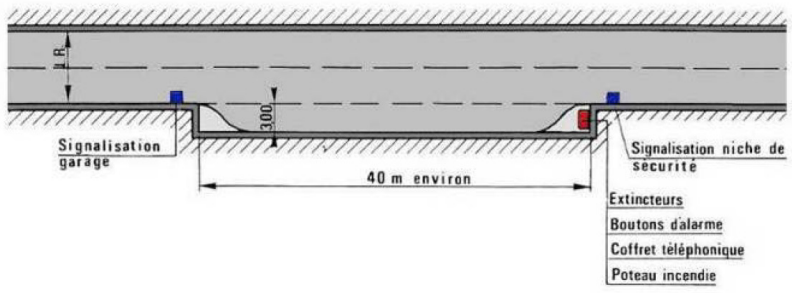

(c)

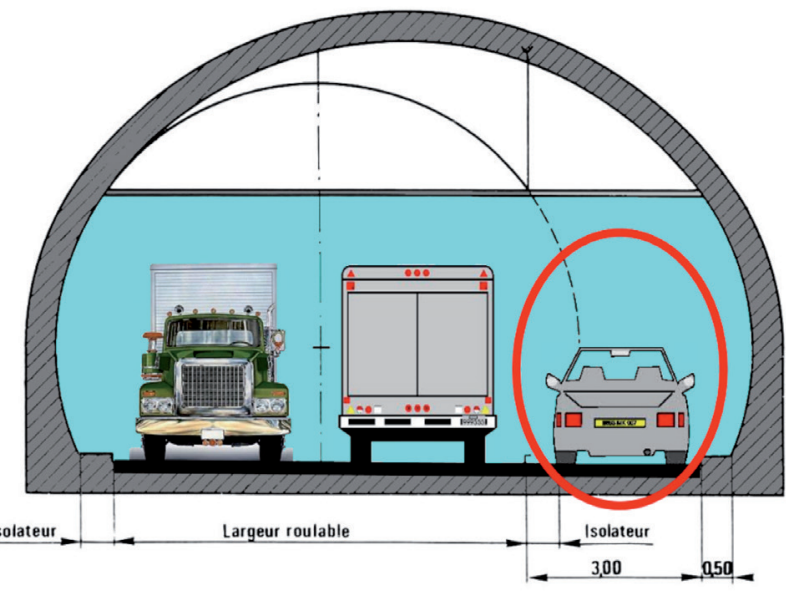

Figura 5: Geometría de los apartaderos, a) planta, b) detalle A y c) sección transversal (CETU, 1976)

\section{Circular Interministerial francesa}

La Circular Interministerial francesa (CIF, 2000) señala que, en túneles de más de $1000 \mathrm{~m}$ de longitud que no dispongan de anchura disponible en el arcén lateral de emergencia para rebasar a un vehículo detenido se construirán apartaderos cada $800 \mathrm{~m}$, distancia que se modulará según las pendientes longitudinales del túnel. Una nueva circular CIF (2006) relativa a la seguridad de túneles de carreteras de longitud superior a $300 \mathrm{~m}$ anula a la CIF (2000) a excepción del Anexo 2, que se mantiene en vigor.

\section{Norma de Austria}

La norma de Austria (RVS 9281, 2002), indica que en túneles unidireccionales de más de $1000 \mathrm{~m}$ se dispondrán los apartaderos en el hastial derecho. En los bidireccionales de longitud mayor de 1000 m estarán ubicados a ambos lados de la calzada, y -si las condiciones geológicogeotécnicas lo permiten- deben disponerse de manera tal que los apartaderos de una y otra calzada queden exactamente enfrentados. La distancia entre los apartaderos consecutivos y la distancia del primer y último al portal será de $1000 \mathrm{~m}$. La longitud de los apartaderos será de 40 $\mathrm{m}$, longitud que deberá aumentarse para la colocación (si se considera necesaria su instalación en el paramento terminal del apartadero) de amortiguadores de impacto (Figura 6). Los teléfonos de emergencia se situarán inmediatamente antes del apartadero según el sentido de avance del tráfico.

(a) $\mathrm{FLN}$
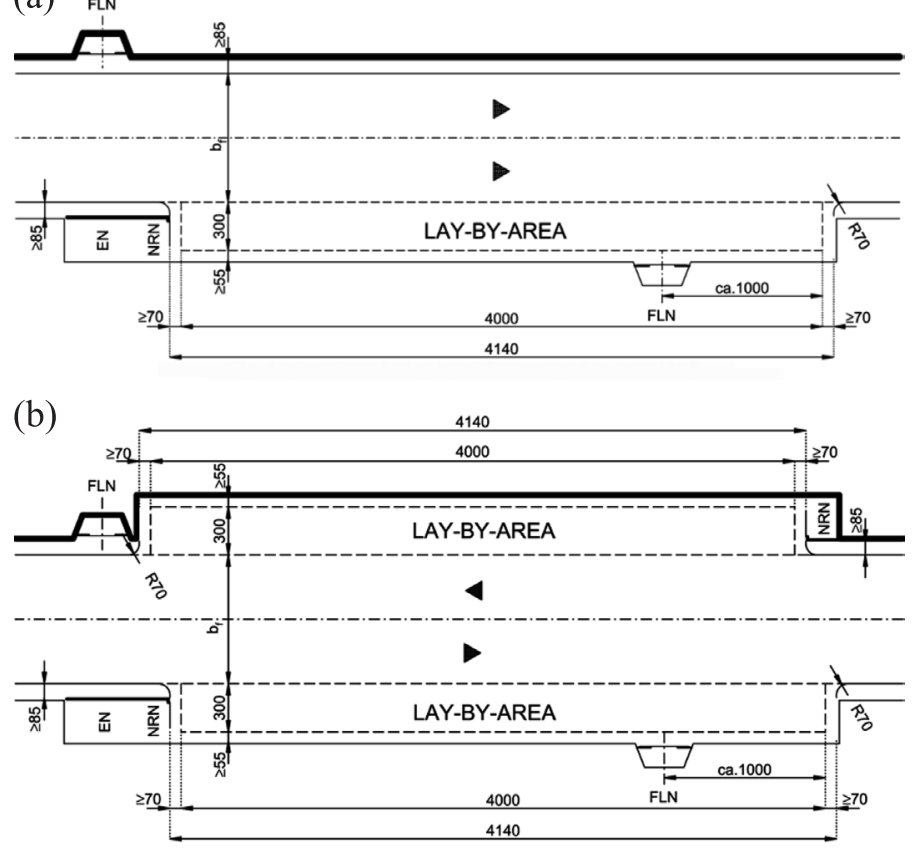

Figura 6: a) Geometría de los apartaderos para túneles unidireccionales y b) bidireccionales. EN: nicho de instalaciones eléctricas, NRN: nicho con teléfono de emergencia y FLN: nicho para extinción de fuego (RVS 9281, 2002)

\section{Norma de Suiza}

La norma de Suiza (SIA 197/2, 2003) señala que en túneles bidireccionales se ubicarán apartaderos, alternativamente, cada 600 a $900 \mathrm{~m}$. En los túneles unidireccionales no se prevé dotarlos de apartaderos excepto aquellos que sean precisos para los locales técnicos que no dispongan de acceso desde el exterior. Si la carretera no dispone de arcenes, en las proximidades a las bocas -en el exterior del túnel- se instalarán apartaderos en ambos sentidos de 
tráfico (si ello es posible). Dichos apartaderos serán del mismo tamaño que los de interior del túnel (Figuras 7 y 8).

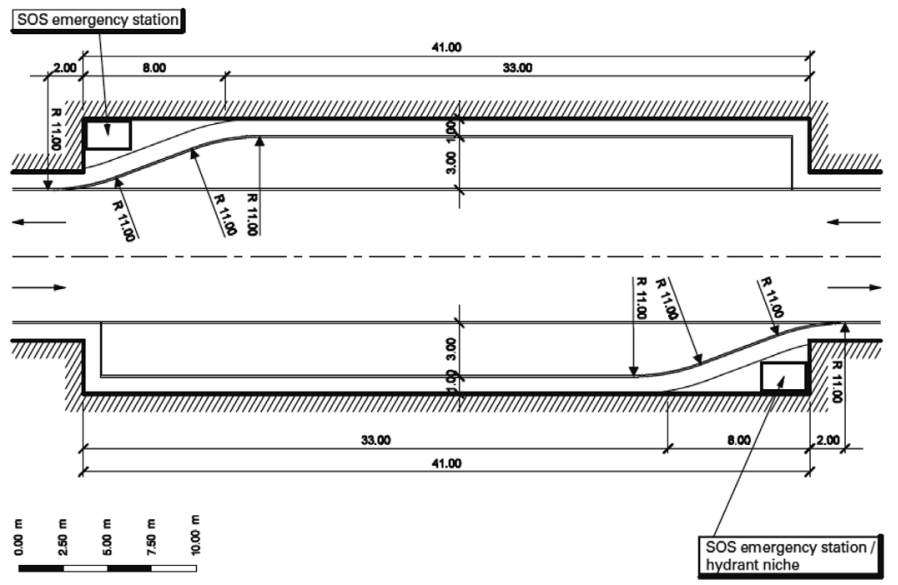

Figura 7: Geometría de los apartaderos según norma Suiza (SIA 197/2, 2003)
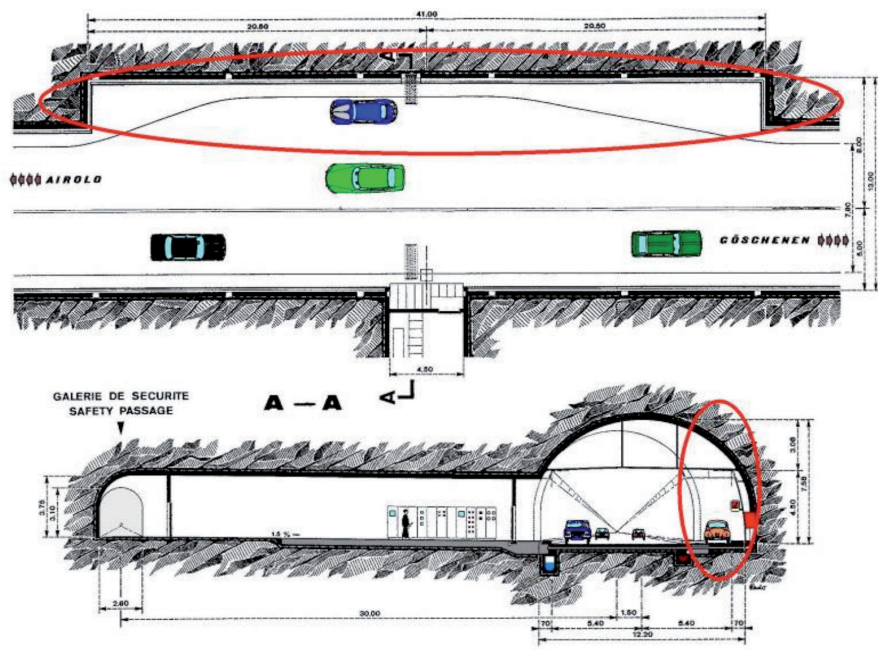

Figura 8: Apartaderos y conexión con la galería de seguridad. Túnel de San Gotardo, Suiza (Lombardi y Haerter, 1972)

\section{Norma de Noruega}

La norma de Noruega (NPRA, 2004) establece las categorías de túneles como punto de partida para el diseño de la sección transversal, número de carriles, necesidad de los apartaderos y galerías de retorno, así como el equipamiento de seguridad. Las categorías de túneles se basan en el volumen de tráfico y en la longitud del túnel. El volumen de tráfico se expresa en AADT (Annual Average Daily Traffic) o tráfico total anual dividido entre 365 considerando ambos sentidos. La categoría del túnel se determina de acuerdo con el AADT estimado 20 años después de la puesta en servicio, AADT(20) (Figura 9). La necesidad y distancia entre apartaderos se establece, como ya se ha dicho, en función de la categoría del túnel. En túneles bidireccionales también se instalarán galerías de retorno para vehículos pesados (los apartaderos también podrán ser empleados para retorno de vehículos ligeros). En túneles largos bidireccionales además se prevé la construcción de galerías de retorno que posibiliten el cambio de sentido para la maquinaria de vialidad invernal (Tabla 1).

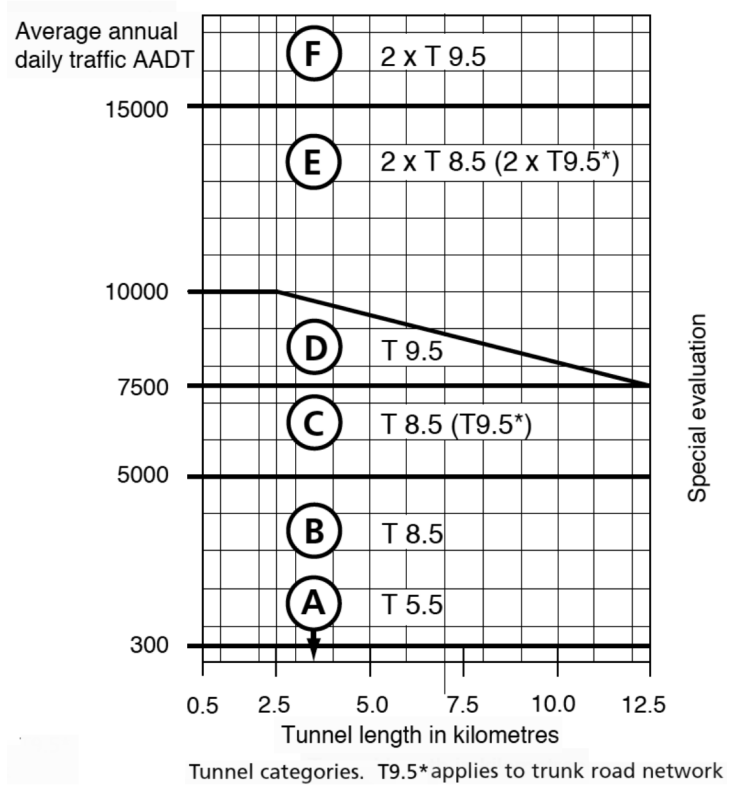

Figura 9: Categoría de Túneles según el AADT(20) y la longitud en km (NPRA, 2004)

Tabla 1: Distancias entre apartaderos y galerías de retorno. Dependiendo de la geología y de la geotecnia estas distancias podrán modificarse hasta $\pm 50 \mathrm{~m}$ en apartaderos $\mathrm{y} \pm 100 \mathrm{~m}$ en galerías de retorno (NPRA, 2004).

\begin{tabular}{|c|c|c|}
\hline $\begin{array}{c}\text { Categoría } \\
\text { del túnel }\end{array}$ & $\begin{array}{c}\text { Distancia entre } \\
\text { apartaderos, } \mathrm{m}\end{array}$ & $\begin{array}{c}\text { Distancia entre } \\
\text { galerías de retorno, } \mathrm{m}\end{array}$ \\
\hline A & - & - \\
\hline B & 500 & 2000 \\
\hline C & 375 & 1500 \\
\hline D & 250 & 1000 \\
\hline E, F & 500 & - \\
\hline
\end{tabular}

La Figura 10 muestra la geometría de tanto los apartaderos como de la galería de retorno que se han comentado. 
(a)
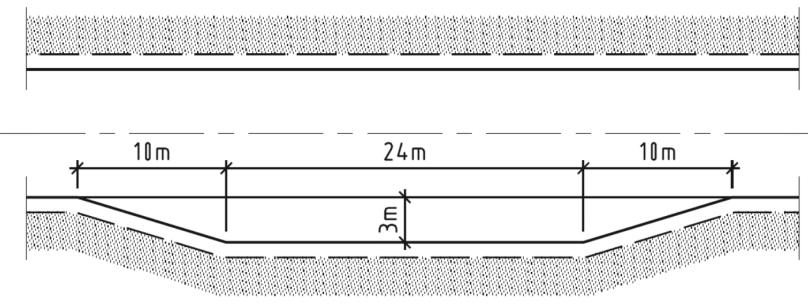

(b)

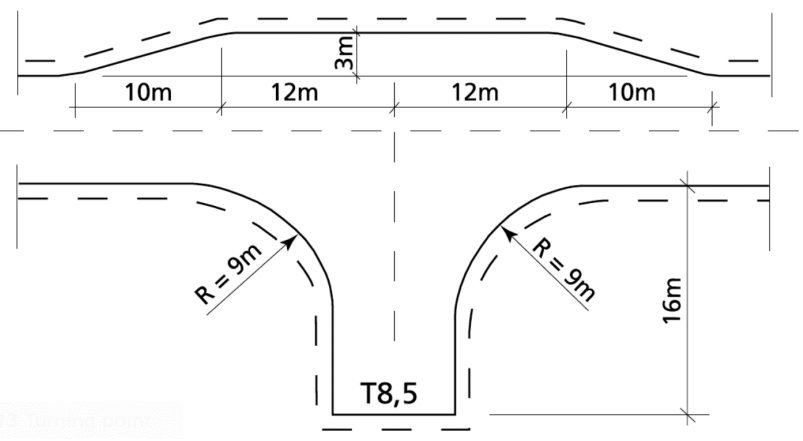

Figura 10: a) Geometría de apartaderos y b) geometría de la galería de retorno para vehículos pesados (NPRA, 2004)

\section{Directiva Europea}

La Directiva Europea (2004) para túneles de la red TransEuropea de carreteras -de más de $500 \mathrm{~m}$ de longitudreferente a los requisitos mínimos de seguridad en los túneles de carreteras para garantizar un nivel mínimo de seguridad en los túneles a los que se aplica, señala para los apartaderos:

\section{Apartaderos:}

- En los túneles bidireccionales en fase de proyecto o construcción de longitud mayor de $1500 \mathrm{~m}$, con un volumen de tráfico superior a 2000 veh/carril, deberán habilitarse apartaderos a distancias no superiores a los $1000 \mathrm{~m}$, caso de que no estén previstos carriles de emergencia o arcenes de anchura superior a $2.5 \mathrm{~m}$.

- En los túneles bidireccionales ya existentes de longitud mayor que $1500 \mathrm{~m}$, con un volumen de tráfico superior a 2000 veh/carril, que no dispongan de carriles de emergencia, se evaluará la viabilidad y eficacia de dotarlos o no de apartaderos mediante los pertinentes análisis de riesgo.

- En los restantes túneles en los que sea requisito la disposición de apartaderos, de acuerdo con el apartado "Equipamiento mínimo según la tipología de túnel
(Túneles unidireccionales de longitud mayor que 1000 m)", cuando las características de la construcción del túnel no lo permitan o sólo lo permitan con costes desproporcionados, no será preciso habilitar apartaderos si la anchura total del túnel accesible para los vehículos, excluyendo las partes elevadas y los carriles normales de circulación, sea al menos igual a la anchura normal de un carril.

- Los apartaderos contarán con un puesto de emergencia.

La Tabla 2 resume lo dicho.

Tabla 2: Necesidad de apartaderos

\begin{tabular}{|c|c|c|}
\hline Tipo de túnel & Longitud y tráfico & Nota \\
\hline $\begin{array}{l}\text { Túneles bidi- } \\
\text { reccionales en } \\
\text { fase de } \\
\text { proyecto o } \\
\text { construcción } \\
\text { y sin carriles de } \\
\text { emergencia o } \\
\text { arcenes de an- } \\
\text { chura }>2.5 \mathrm{~m}\end{array}$ & $\begin{array}{c}\mathrm{L}>1500 \mathrm{~m} \text { y } \\
\text { volumen de tráfico } \\
>2000 \text { veh/carril } \\
\text { Distancia entre aparta- } \\
\text { deros } \leq 1000 \mathrm{~m}\end{array}$ & \\
\hline $\begin{array}{l}\text { Túneles bidirec- } \\
\text { cionales } \\
\text { existentes } \\
\text { y sin carriles de } \\
\text { emergencia }\end{array}$ & $\begin{array}{c}\mathrm{L}>1500 \mathrm{~m} \text { y } \\
\text { volumen de tráfico }> \\
2000 \text { veh/carril } \\
\text { Distancia entre aparta- } \\
\text { deros } \leq 1000 \mathrm{~m}\end{array}$ & $\begin{array}{l}\text { Se evalúa la via- } \\
\text { bilidad y eficacia } \\
\text { según análisis de } \\
\text { riesgo }\end{array}$ \\
\hline $\begin{array}{l}\text { Túneles unidi- } \\
\text { reccionales }\end{array}$ & $\begin{array}{c}\mathrm{L}>1500 \mathrm{~m} \\
\text { Distancia entre aparta- } \\
\text { deros } \leq 1000 \mathrm{~m}\end{array}$ & $\begin{array}{l}\text { Cuando las caracte } \\
\text { rísticas de la cons } \\
\text { trucción del túne } \\
\text { no lo permitan } \\
\text { sólo lo permitan } \\
\text { con costes despro- } \\
\text { porcionados, no } \\
\text { será preciso habi } \\
\text { litar apartaderos } \\
\text { si la anchura tota } \\
\text { del túnel accesible } \\
\text { para los vehículos } \\
\text { excluyendo las par } \\
\text { tes elevadas y los } \\
\text { carriles normales } \\
\text { de circulación, sea } \\
\text { al menos igual a la } \\
\text { anchura normal de } \\
\text { un carril. }\end{array}$ \\
\hline
\end{tabular}


Si alguno de esos requisitos que establece la Directiva Europea no fuera satisfecho en un túnel, la propia Directiva establece un procedimiento para lograr la aceptación de esa desviación. No obstante, es importante anotar que en el caso de los apartaderos no se acepta desviación alguna. La Directiva Europea puntualiza que no se permitirá desviación alguna de los requisitos establecidos por la propia Directiva en los siguientes elementos de seguridad: emisoras de emergencia, señales, apartaderos, salidas de emergencia, y retransmisión por radio.

\section{Algunos problemas relacionados con los apartaderos}

Si bien los apartaderos en túneles de carretera pueden ser de ayuda en determinados casos, también presentan algunos aspectos sobre los que conviene reflexionar. El apartado que ahora nos ocupa se centrará en la presentación de algunos accidentes en los apartaderos. Es importante prestar atención en los paramentos extremos de los apartaderos para evitar que ofrezcan una cara perpendicular al sentido de la circulación pues un impacto de un vehículo sería una colisión frontal o más o menos descentrada, es decir, cuando no todo el frontal del vehículo choca contra el objeto, sino que la colisión se concentra en una porción de éste contra un muro y podría tener consecuencias de importancia. A continuación se presentan casos de accidentes recientes en los que un vehículo colisionó contra los extremos de apartaderos.

\section{Túnel de Sierre}

El 13 de marzo de 2012, en el túnel suizo de Sierre (Autopista A-9, cerca de Sierre, Cantón de Valais, Francia), un autocar pierde el control e impacta contra el muro del extremo final de uno de los apartaderos. En el autobús viajaban 52 personas: fallecieron 22 niños y 6 adultos, 24 niños resultaron heridos. Se trata de un túnel unidireccional, de $2460 \mathrm{~m}$ de longitud, inaugurado en 1999, proyectado según las actuales normativas, disponiendo entre otros elementos de seguridad de salidas de emergencia cada 300 $\mathrm{m}$, extintores y teléfonos de emergencia cada $150 \mathrm{~m}, \mathrm{y}$ apartaderos cada $600 \mathrm{~m}$ (Figuras 11 y 12).

\section{Accidentes en España}

Los muros extremos finales de los apartaderos han influido en mayor o menor medida en el resultado de los accidentes.
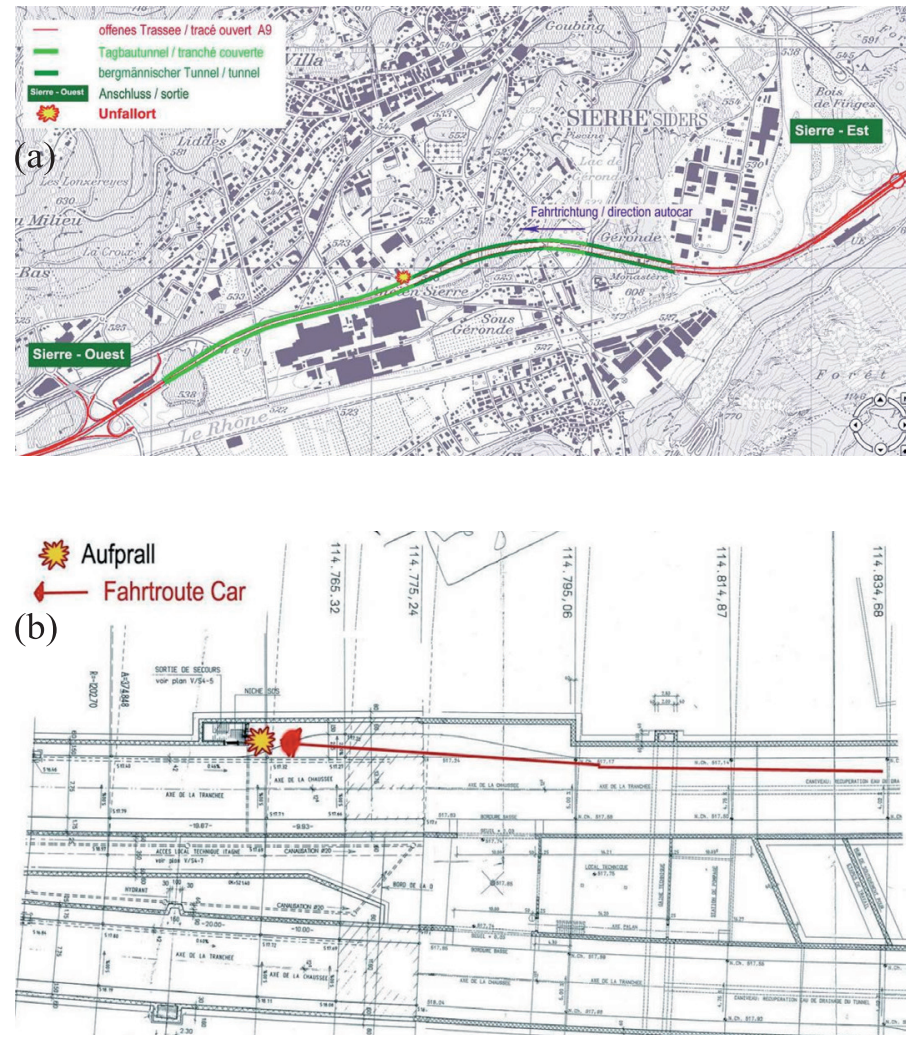

Figura 11: Túnel de Sierre, Suiza, a) planta general del túnel y b) planta de detalle del apartadero (FEDRO, 2012)
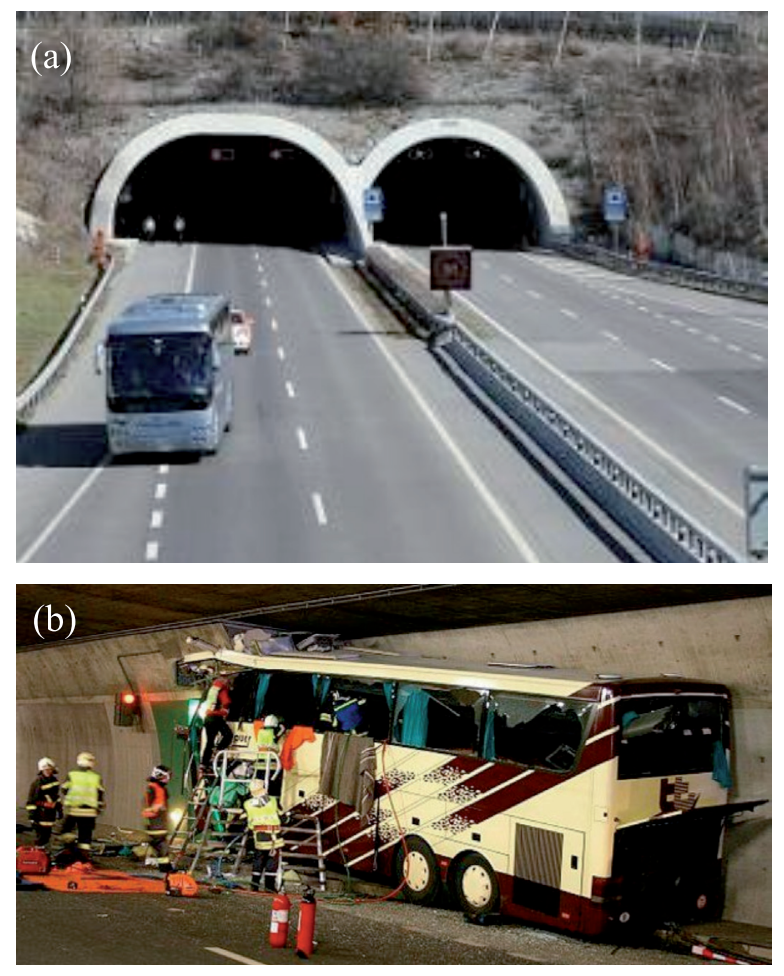

Figura 12: Túnel de Sierre, Suiza, a) vista de las bocas y b) accidente con colisión de un autobús con el final de un apartadero (fuente: Excite Network) 
Los tres casos de accidentes han sido en los siguientes túneles:

i) Túnel de El Perdón, Autovía del Camino, A-12, Navarra, de $1170 \mathrm{~m}$ de longitud, 15 de enero de 2010, 1 herido (Figura 13). iia) Túnel de Priañes, Autovía A-63, Asturias, de 620 m de longitud: 1 de febrero de 2012 (Noticias de Grao, Periódico La Voz de Asturias), falleció el conductor del vehículo. iib) Túnel de Priañes, 18 de septiembre de 2014 (elcomercio.es), una furgoneta colisionó por detrás contra un turismo (Renault Clio), éste perdió el control y chocó contra el hastial en el borde del muro final del apartadero, fallecieron los 2 ocupantes del turismo.

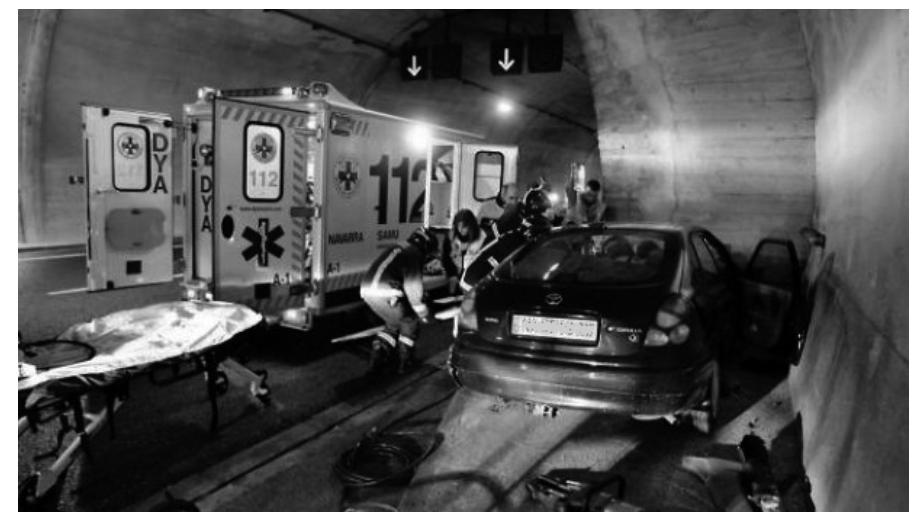

Figura 13: Túneles de El Perdón. Colisión de un vehículo contra el final de un apartadero, 15 enero 2010 (fuente: j.bergasa. noticiasdenavarra.com)

\section{Estudios en la República de Slovenia}

Según el estudio de Sajovic (2014), en la República de Slovenia, desde el año 2010 hasta octubre del 2014, 13 personas murieron en impactos contra los muros finales de los apartaderos. Los conductores circulaban solos en sus vehículos, excepto en un caso. Sólo en dos casos se confirmó el suicidio. El número de túneles era 21, el número de túneles con apartaderos 6, el número de apartaderos 24 y la velocidad máxima en los túneles $100 \mathrm{~km} / \mathrm{h}$. A partir del análisis de las imágenes de video, se establece las trayectorias de los vehículos que colisionaron. Por otro lado, Sajovic (2014) señala que en un apartadero de 40 $\mathrm{m}$ de longitud, el mínimo necesario para las maniobras de entrada y salida de un vehículo pesado con remolque es de $36 \mathrm{~m}$, habiendo por lo tanto una disponibilidad para instalar los sistemas de protección de $4 \mathrm{~m}$.

El autor citado informa de la modelización computacional llevada a cabo por la Facultad de Ingeniería Mecánica de la Universidad de Ljubljana (Slovenia), habiéndose simulado conforme a la Norma UNE EN 1317-3 (2011) tres tipos de defensas: i) barrera de contención metálica de $4 \mathrm{~m}$ de longitud, ii) barrera de contención metálica de $8 \mathrm{~m}$ longitud y iii) atenuador de impacto modificado de 4 $\mathrm{m}$ de longitud y $2 \mathrm{~m}$ de ancho. El estudio concluye que para los túneles existentes la solución más favorable es la que emplea el atenuador de impacto modificado: pero para obtener el Certificado $\mathrm{CE}$ se han de realizar ensayos físicos de choque (ver Figura 14).

a)

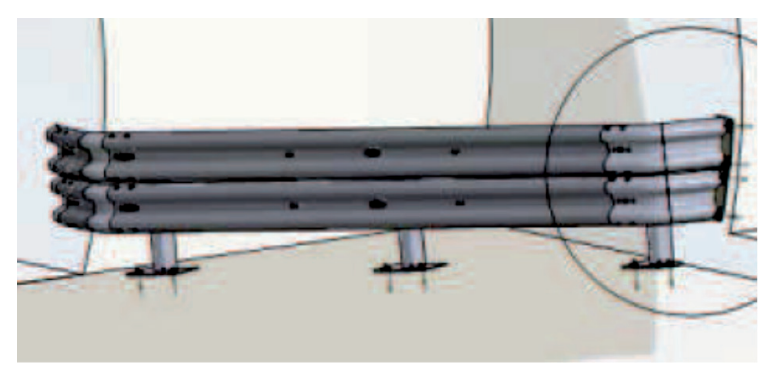

b)

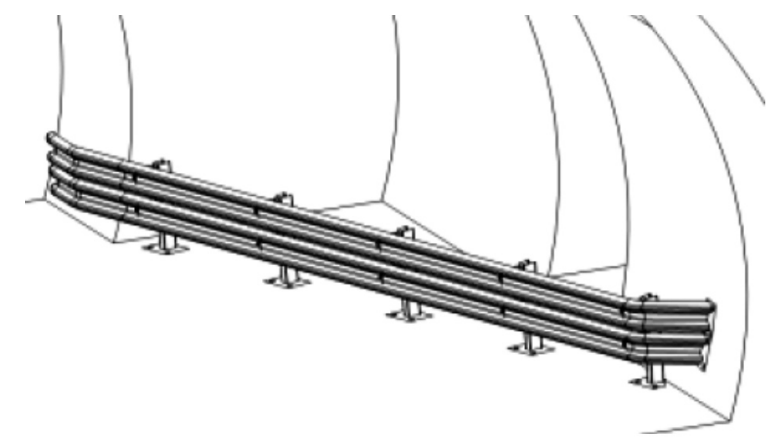

c)
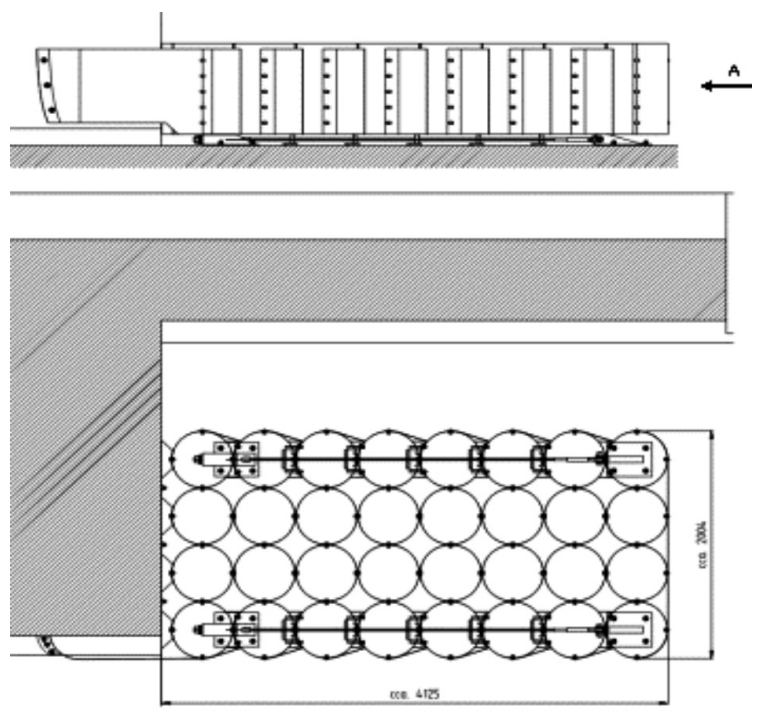

Figura 14: Barrera de contención metálica de a) $4 \mathrm{~m}$ y b) $8 \mathrm{~m}$ de longitud y c) atenuador de impacto modificado de $4 \mathrm{~m}$ de longitud y $2 \mathrm{~m}$ de ancho (Sajovic, 2014) 


\section{Conclusiones}

Se presentan antecedentes de accidentes relacionados con apartaderos en túneles de carreteras, los cuales dan a conocer posibles problemas en los diseños de los paramentos extremos de los mismos. Es muy frecuente construir dichos extremos perpendiculares al sentido del tráfico. En aquellos casos en los que los extremos se construyen oblicuos al sentido del tráfico solían serlo más por razones de trazado en la idea de facilitar a los usuarios tanto las entradas como las salidas a los mismos, y no tanto por razones de seguridad frente a impactos: en algunos casos un bordillo y/o cebreado marca el trazado de entrada y salida a los mismos.

El grave accidente del túnel suizo de Sierre -así como otros habidos en otros túneles con vehículos ligerosaconsejan reflexionar al respecto de aquellos factores que hayan podido influir o agravar el accidente, así como en las posibles medidas a tomar para evitarlo -0 mitigar sus efectos- en un futuro. Parece que la existencia de paredes perpendiculares y muy posiblemente también las que se construyen con cierto ángulo estricto al sentido del tráfico y sin protección de los apartaderos deben ser consideradas peligrosas. Este peligro es especialmente relevante en el espacio confinado de un túnel, un peligro que se acentúa por el hecho de que sólo la más mínima desviación de la trayectoria del conductor a la derecha (la existencia de apartaderos está asociada en general a la existencia de un arcén insuficiente) si es que ocurre exactamente en el momento equivocado, podría dar lugar a un impacto severo en el muro extremo del apartadero.

Tal vez la protección para mitigar los daños en caso de impacto en túneles existentes puedan diseñarse empleando elementos tales como barreras de seguridad metálicas, barreras de hormigón y atenuadores de impacto. Las soluciones no son evidentes y han de ser estudiadas con rigor -y para el caso específico del objeto de estudiopara que sean realmente eficaces. En esos estudios han de involucrarse expertos en sistemas de contención de vehículos, así como deben realizarse ensayos físicos a escala real.

También debe reflexionarse el uso real de esos apartaderos. En proyectos nuevos debe evaluarse la conveniencia de dotar a los túneles de anchura suficiente en la plataforma de rodadura para que no sean necesarios los apartaderos. En los nuevos proyectos tal vez sea aconsejable diseñar los extremos finales de los apartaderos con ángulos mucho más suaves, del orden de 1:20 respecto a dirección del carril derecho de la calzada. En túneles existentes construidos con apartaderos debería reflexionarse sobre la necesidad de tener operativos dichos apartaderos: el índice de utilización de los apartaderos por vehículos averiados es bastante bajo: tan sólo el 20\% (AIPCR, 2011).

\section{Referencias}

AIPCR (2011). Manual de Túneles de Carretera. 7. Obras específicas necesarias para la explotación y seguridad. 7.2. Obras destinadas a los vehículos. Asociación Mundial de Carreteras. http://tunnels.piarc.org/es/preambulo/

CETU (1976). Dossier Pilote des Tunnels. Ministere de l'Equipement du Logement. CETU: Centre d'Etudes des Tunnels, Lyon, France

CIF (2000). Circulaire Interministerielle No 2000-63 DU relative à la sécurité dans les tunnels du réseau routier national. Ministere de l'Interieur; Ministere de l'Equipement des Transports et du Logement

CIF (2006). Circulaire Interministerielle $\mathrm{N}^{\circ}$ 2006-20. Annexe $\mathrm{n}^{\circ} 2$. Instruction technique relative aux dispositions de securite dans les nouveaux tunnels routiers: Conception et Exploitation. Ministere de l'Interieur; Ministere de l'Equipement des Transports et du Logement

Directiva Europea (2004). Requisitos mínimos de seguridad para túneles de la red Europea de carreteras. Directiva 2004/54/ CE del Parlamento Europeo y del Consejo de la Unión Europea. Diario Oficial de la Unión Europea

DOKA (2007). Encofrados para túneles. Jornada técnica austríaca de construcción de túneles y tecnología de ferrocarril. Oficina Comercial de la Embajada de Austria. 6 de marzo 2007

FEDRO (2012). Fatal coach crash in the Sierre tunnel on 13 March 2012. The Swiss Federal Roads Office.

Lombardi, G. et Haerter, A. (1972). N2 Le tunnel routier du StGothard: Le projet du tunnel. Service Topographique Fédéral

MAFRA, s.a. (2014). Zabedněný a opuštěný. Prokletý tunel Blanka mîrí před soud. 2 de octubre de 2014 (en checo) 
Norma UNE-EN 1317-3 (2011). Sistemas de contención para carreteras. Parte 3: Clases de comportamiento, criterios de aceptación para el ensayo de impacto y métodos de ensayo para atenuadores de impactos.

NPRA (2004). Road Tunnels. Manual 021. Norwegian Public Roads Administration.

RVS 9281 (2002). Planning guidelines. Operating and safety facilities constructions. Bearbeitet von der österreichischen Forschungsgemeinschaft Straße und Verkehr FSV, Arbeitsgruppe, Tunnelbau, Arbeitsausschuss, Betriebs- und Sicherheitseinrichtungen, Austria

Sajovic, J. (2014). The Technology of Highway Safety. Lay-bys and protection against lateral obstacles: Situation in Slovenia. 2014 IBTTA Global Summit. Innovations \& Technologies for Sustainable Mobility, Environment and Road Safety. Prague, Czech Republic. October 19-21, 2014.
Samaniego, A., Felix, O. y Soldi, C. (2008). Estudios de Ingeniería del Túnel Marcavalle en la Vía de Evitamiento la Oroya, Perú. $2^{\circ}$ Congreso Brasilero de Túneles y Estructuras Subterráneas. Seminario Internacional South American Tunnelling (SAT'2008). São Paulo, Brasil, 23-25 junio 2008.

SIA 197/2 (2003). Design of Tunnels: Road Tunnels. Schweizer Norm. Swiss Society of Engineers and Architects. Zurich, Switzerland. 